\title{
DESENVOLVIMENTO DE MODELO PARA PREVER A EFICIÊNCIA DE MISTURAS DESSULFURANTES DE FERRO GUSA ATRAVÉS DE TERMODINÂMICA COMPUTACIONAL*
}

Sábata Marla Reis Durães Oliveira ${ }^{1}$ Heitor Cristo Clem de Oliveira² Silas Gambarine Soares² Felipe Fardin Grillo ${ }^{3}$ Jorge Alberto Soares Tenorio ${ }^{4}$

Resumo José Roberto de Oliveira

Este trabalho tem como objetivo o uso de modelos termodinâmicos da literatura e termodinâmica computacional no estudo de dessulfuração de ferro-gusa através de misturas $\mathrm{CaO}$-Fluorita e $\mathrm{CaO}$-Sodalita. As experiências foram realizadas com a adição de diferentes misturas destes sistemas em ferro-gusa a uma temperatura de $1400^{\circ} \mathrm{C}$. Os resultados dos testes foram analisados e comparados com os obtidos pelos modelos termodinâmicos da literatura e o software de termodinâmica computacional ThermoCalc. Através dos modelos da literatura foram calculados basicidade óptica, capacidade de sulfeto (Cs) e partição de enxofre (Ls). Pelo software ThermoCalc foram calculados o teor de enxofre de equilíbrio no metal, fases sólidas, e a quantidade de líquido em cada uma das misturas dessulfurantes utilizadas no processo a uma temperatura de $1.400^{\circ} \mathrm{C}$. O ThermoCalc mostrou-se mais preciso que os modelo da literatura na análise da capacidade de dessulfuração das misturas utilizadas neste trabalho.

Palavras-chave: Ferro-gusa; Dessulfuração; Termodinâmica computacional; Modelos termodinâmicos.

\section{DEVELOPMENT OF A MODEL FOR PREDICTING THE HOT METAL DESULFURIZING MIXTURES EFFICIENCY BY COMPUTATIONAL THERMODYNAMICS}

\section{Abstract}

This work aims at the use of literature models thermodynamics and computational thermodynamics in the study of hot metal desulfurization through $\mathrm{CaO}$-Fluorspar and $\mathrm{CaO}$-Sodalite mixtures. Experiments were performed with the addition of different mixtures from these systems in molten hot metal at a temperature of $1,400^{\circ} \mathrm{C}$. The test results were analyzed and compared with those obtained by the literature models thermodynamics and computational thermodynamics software program ThermoCalc. Through literature models were calculated optical basicity, sulfide capacity (Cs) and sulfur partition (Ls). By software ThermoCalc were calculated the equilibrium sulfur content in the metal, solid phases, and the amount of liquid in each desulfurizing mixture used in the process at a temperature of $1,400^{\circ} \mathrm{C}$. ThermoCalc showed up more accurate than literature model of $\mathrm{Cs}$ and $\mathrm{Ls}$ in analysis of desulfurizing efficiency of the mixtures used in this paper.

Keywords: Hot metal; Desulfurization; Computational thermodynamics; Models.

Eng. Metalurgista, Doutorando em Engenharia Metalúrgica e de Materiais, PRORPEMM, IFES, Vitoria, ES, Brasil.

2 Eng. Metalurgista, Mestrando em Engenharia Metalúrgica e de Materiais, PRORPEMM, IFES, Vitoria, ES, Brasil.

Doutorando em Engenharia Metalúrgica e de Materiais pela USP, São Paulo, SP, Brasil.

Prof. Titular do Departamento de Química da USP, São Paulo, SP, Brasil.

Prof. Dr., cursos de graduação e pós-graduação em Engenharia Metalúrgica e de Materiais, Instituto Federal do Espírito Santo (IFES), Vitoria, ES, Brasil. 


\section{INTRODUÇÃO}

O principal agente dessulfurante utilizado industrialmente é a cal (CaO). A reação de dessulfuração do ferro-gusa pode ocorrer de acordo com a reação $[1,2]$.

$$
[S]+\mathrm{CaO}_{(S)}=\mathrm{CaS}_{(S)}+[\mathrm{O}]
$$

Dependendo do teor de carbono e silício do metal e de $\mathrm{SiO}_{2}$ e $\mathrm{Al}_{2} \mathrm{O}_{3}$ da escória podem ocorrer as seguintes reações $[2,3]$.

$$
\begin{array}{r}
{[\mathrm{S}]+[\mathrm{C}]+\mathrm{CaO}_{(S)}=\mathrm{CaS}_{(S)}+\mathrm{CO}_{(g)}} \\
5 \mathrm{CaO}_{s}+2[\mathrm{~S}]+[\mathrm{Si}]+=2 \mathrm{CaS}_{(S)}+3 \mathrm{CaO} \cdot \mathrm{SiO}_{2(S)} \\
5 \mathrm{CaO}_{s}+2[\mathrm{~S}]+\mathrm{SiO}_{2(S)}=2 \mathrm{CaS}_{(S)}+3 \mathrm{CaO} \cdot \mathrm{SiO}_{2(S)} \\
3 \mathrm{CaO}_{s}+\mathrm{SiO}_{2(s)}=\mathrm{Ca}_{3} \mathrm{SiO}_{2(s)} \\
3 \mathrm{CaO}_{(s)}+\mathrm{Al}_{2} \mathrm{O}_{3(\mathrm{~S})}=\mathrm{Ca}_{3} \mathrm{Al}_{2} \mathrm{O}_{6(s)}
\end{array}
$$

Conforme mostrado nas reações 1, 3 e 5, a reação entre o enxofre e a cal forma 0 sulfeto de cálcio (CaS), o qual se forma em torno da partícula de $\mathrm{CaO}$. Segundo Ohya [4], quando $15 \%$ da partícula de $\mathrm{CaO}$ é convertido em $\mathrm{CaS}$, o poder dessulfurante do $\mathrm{CaO}$ é praticamente extinguido. Além do $\mathrm{CaS}$, existem outros compostos que reagem com a partícula de $\mathrm{CaO}$ formando compostos de cálcio, entre estes, pode-se destacar os silicatos de cálcio $\left(2 \mathrm{CaO} \mathrm{SiO}_{2}\right.$ e $\left.3 \mathrm{CaO} . \mathrm{SiO}_{2}\right)$ e 0 aluminato de cálcio $\left(3 \mathrm{CaO} \cdot \mathrm{Al}_{2} \mathrm{O}_{3}\right)$. Segundo Mitsuo et al [5], estes compostos possuem um alto ponto de fusão e se formam ao redor da partícula de $\mathrm{CaO}$, impedindo a transferência dos íons de $\mathrm{S}^{-2}$ e limitando a reação de dessulfuração.

Tendo em vista o fenômeno supracitado, é comum a adição de agentes fluxantes como a fluorita $\left(\mathrm{CaF}_{2}\right)$ na mistura dessulfurante, os quais tem a função de dissolver os compostos sólidos formados ao redor da partícula de $\mathrm{CaO}$. Segundo Niedringhaus e Fruehan [6], o $\mathrm{CaF}_{2}$ possui efeito significante na mistura até uma concentração máxima de $10 \%$.

Para avaliar a eficiência de remoção de enxofre do ferro gusa por uma dada mistura, são normalmente utilizados os seguintes parâmetros: basicidade óptica $(\Lambda)$, capacidade sulfureto (Cs) e coeficiente de partição de enxofre (Ls). Sosinsky e Sommerville [7] determinaram valores de basicidade óptica para alguns elementos que são tipicamente utilizados em misturas dessulfurantes, a saber: $\mathrm{CaO}$ $=1 ; \mathrm{MgO}=0,78 ; \mathrm{CaF} 2=0,37 ; \mathrm{SiO} 2=0,48 ; \mathrm{Al} 2 \mathrm{O} 3=0,61 ; \mathrm{P} 2 \mathrm{O} 5=0,40 ; \mathrm{Na} 2 \mathrm{O}=$ 1,15. Young [8], definiu a capacidade de sulfeto como a capacidade da escória de remover enxofre, estabelecendo relações entre Cs e basicidade ótica, mostradas pelas equações (6) e (7).

$$
\begin{aligned}
& \log C_{S}=-13,913+42,84 \Lambda-23,82 \Lambda^{2}-\frac{11710}{T}-0,02223 . \% \mathrm{SiO}_{2} \\
& -0,02275 . \% \mathrm{Al}_{2} \mathrm{O}_{3} \\
& \quad \log C_{S}=-0,6261+0,4808 \Lambda+0,7917 \Lambda^{2}+\frac{1697}{T}-\frac{2587 \Lambda}{T} \\
& \quad+0,0005144 . \% \mathrm{FeO}
\end{aligned}
$$

A equação (6) é válida para as escórias com $\Lambda<0,8$ e a equação (7) é válida para as escórias com $\Lambda \geq 0,8$.

O coeficiente de partição do enxofre é um parâmetro que exprime a relação entre a concentração de enxofre na escória (\%S eq. $)$ e no banho metálico [\% $\mathrm{S}_{\text {eq. }}$. mediante o 
equilíbrio termodinâmico [7]. Inoue e Suito [9] proporam o modelo de Ls, que é função do $\mathrm{C}_{\mathrm{s}}$, temperatura e elementos dissolvidos no ferro gusa.

$$
\log L_{S}=\log C_{S}-\frac{1053}{T}+5,73+\log f_{S}
$$

Além dos modelos citados, existem outros como Yoshinori et al [10]. Porém todos estes modelos apresentam resultados diferentes, que nem sempre são consistentes com aqueles encontrados na prática. Além disso, estes modelos não fornecem informações sobre viscosidade, quantidade de líquidos e sólidos, e as fases presentes nas misturas e, consequentemente, sobre a cinética da dessulfuração. Por isso, estes modelos podem levar a erros quando se tenta prever a eficiencia de dessulfuração de misturas através deles. O conhecimento dos parâmetros que influenciam a cinética de dessulfuração (fases presente, a percentagem de líquido e sólido e viscosidade) é da maior importância, uma vez que muitas vezes o limite para a obtenção de níveis mais baixos de enxofre em processos industriais, não é termodinâmico, mas cinético.

A velocidade de dessulfuração é controlada pela difusão do enxofre até a camada de $\mathrm{CaO}$ e pode ser descrita pela equação 8 [6].

$$
-\frac{d[S]}{d t}=\frac{A}{V} \cdot k_{m} \cdot\left([S]-\left[S_{e}\right]\right)
$$

Onde:

$A$ : área da interface entre $\mathrm{CaO}$ e metal $\mathrm{m}, \mathrm{km}_{\mathrm{m}}$ coeficiente de transferência de massa transfer rate in metal $(\mathrm{m} / \mathrm{min}), V$ : volume do metal $\left(\mathrm{m}^{3}\right), K$ : constante de velocidade $(1 / \mathrm{min})$.

Como mostrado nas equações 3 e 4 a reação de DeS pode gerar diferentes fases solidas que podem se formar em torno das partículas de $\mathrm{CaO}$ diminuído a área da interface entre o $\mathrm{CaO}$ e o metal, diminuindo consequentemente a eficiência de dessulfuração [6].

Estes autores mostram que $5 \%$ de $\mathrm{CaF}_{2}$ a $1450^{\circ} \mathrm{C}$ forma $20 \%$ de fase líquida, a qual é suficiente para evitar a precipitação das fases sólidas silicato tricálcico $\left(\mathrm{Ca}_{3} \mathrm{SiO}_{5}\right)$, CaS, $\mathrm{Ca}_{3} \mathrm{AlO}_{5}$.

Estes resultados reforçam o fato de que o conhecimento das fases presentes e a percentagem de líquido e sólido na mistura desulfurante é fundamental para a compreensão do processo. A termodinâmica computacional é uma ferramenta que permite a determinação destes parâmetros, e também uma previsão de qual a mistura vai ser mais eficiente em processos industriais.

\section{MATERIAIS E MÉTODOS}

\subsection{Determinação das Misturas}

Para a realização deste trabalho, inicialmente foi definida a composição química das misturas dessulfurantes. A composição química das misturas usadas em porcentagem é mostrada na Tabela 1 e em massa na Tabela 2. Inicialmente foi preparada 10 gramas de cada mistura. Porém quando estas misturas entram em contato com o banho, ocorrem as perdas por calcinação do material volátil, resultando em uma massa menor. 
Tabela 1 Composição química em porcentagem massa das misturas dessulfurantes propostas.

\begin{tabular}{ccccccc}
\hline \multirow{2}{*}{ Misturas } & \multicolumn{5}{c}{ Composição química (\%) } & Massa de \\
\cline { 2 - 5 } & $\mathbf{C a O}$ & $\mathbf{N a}_{2} \mathbf{O}$ & $\mathbf{C a F}_{2}$ & $\mathbf{A l}_{2} \mathbf{O}_{3}$ & $\mathbf{S i O}_{2}$ & Mistura(g) \\
\hline F5 & 92,50 & - & 4,70 & 0,42 & 2,49 & 9,26 \\
F5- S & 89,35 & - & 4,70 & 0,417 & 5,50 & 9,58 \\
F10 & 87,43 & - & 9,16 & 0,61 & 2,80 & 9,28 \\
F10- S & 84,12 & - & 9,13 & 0,62 & 5,80 & 9,64 \\
N1 & 92,95 & 0,65 & - & 1,50 & 4,90 & 9,21 \\
N2 & 88,38 & 1,30 & - & 2,70 & 7,62 & 9,19 \\
\hline
\end{tabular}

Como pode ser observado na Tabela 1 foi usado misturas com diferentes teores de $\mathrm{CaF}_{2}$, não, $\mathrm{SiO}_{2}$ e $\mathrm{Al}_{2} \mathrm{O}_{3}$, com teores que são próxima os dos usados industrialmente.

As misturas de $\mathrm{F} 5$ e $\mathrm{F} 10$ foram feitas com adição Fluorita da seguinte forma:

- 5 \% (mistura F5) e 10\% (mistura F10), enquanto as misturas F5-S e F10-S tiveram adição de 3\% $\mathrm{De}^{\mathrm{SiO}} 2$ nas misturas $\mathrm{F} 5$ e $\mathrm{F} 10$.

Tabela 2. Composição química em massa e massa das misturas dessulfurantes propostas

\begin{tabular}{|c|c|c|c|c|c|c|}
\hline \multicolumn{6}{|c|}{ Composição Química (g) } & \multirow{2}{*}{$\begin{array}{l}\text { Massa da } \\
\text { mistura(g) }\end{array}$} \\
\hline Mixtura & $\mathrm{CaO}$ & $\mathrm{Na}_{2} \mathrm{O}$ & $\mathrm{CaF}_{2}$ & $\mathrm{Al}_{2} \mathrm{O}_{3}$ & $\mathrm{SiO}_{2}$ & \\
\hline F5 & 8,56 & - & 0,43 & 0,04 & 0,23 & 9,26 \\
\hline F5- S & 8,56 & - & 0,45 & 0,04 & 0,53 & 9,58 \\
\hline F10 & 8,11 & - & 0,85 & 0,06 & 0,26 & 9,28 \\
\hline F10-S & 8,11 & - & 0,88 & 0,06 & 0,56 & 9,64 \\
\hline N1 & 8,56 & 0,06 & - & 0,14 & 0,45 & 9,21 \\
\hline N2 & 8,12 & 0,12 & - & 0,25 & 0,70 & 9,19 \\
\hline
\end{tabular}

Após a determinação da composição química das misturas a serem utilizadas, foram realizados os experimentos, os cálculos termodinâmicos através dos modelos presentes na literatura $\left(\Lambda, C_{s}\right.$ e Ls) e simulações termodinâmicas através do ThermoCalc v.5 e FactSage 6.2.

\subsection{Procedimento Experimental}

Inicialmente, ferro-gusa sólido foi carregado em cadinhos de $\mathrm{MgO}-\mathrm{C}$ e inserido em um forno de resistência elétrica MAITEC, modelo $1700-\mathrm{FEE}$ a $1400^{\circ} \mathrm{C}$. Além disso, gás argônio foi soprado sobre o ferro-gusa a uma taxa de $10 \mathrm{Nl} / \mathrm{min}$, a fim de inertizar o ambiente e evitar a oxidação do metal.

Depois de aproximadamente 40 min., o ferro-gusa estava totalmente fundido. Então, foi retirada uma amostra inicial do metal através de um amostrador à vácuo e, em seguida, a mistura foi adicionada sobre o ferro-gusa através de um tubo de aço inoxidável. Depois de adicionada a mistura, um impeller de alumina foi inserido no banho a uma rotação de $400 \mathrm{rpm}$. Amostras foram retiradas nos tempos de 10, 15, 20 e 30 minutos. A Figura 1 mostra um diagrama esquemático dos experimentos realizados. 


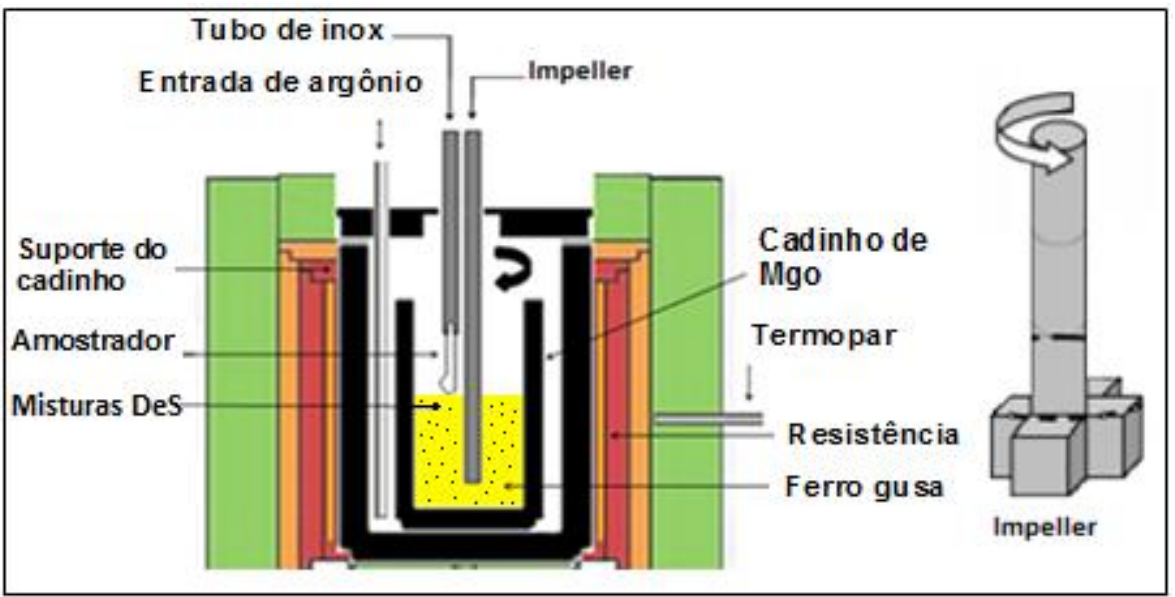

Figura 1 Diagrama esquemático dos experimentos realizados.

\subsection{Avaliação Termodinâmica das Misturas}

Primeiro, as misturas dessulfurantes foram avaliadas termodinamicamente segundo a capacidade de sulfeto (Cs) e a partição de enxofre (Ls). Além disso, através das composições químicas das misturas e do ferro-gusa, simulações termodinâmicas foram realizadas utilizando o software Thermocalc TCW V.5, e o banco de dados Slag3. Para determinar as fases presentes e quantidade de líquido e de solido nas diferentes misturas, foi simulado através do Thermocalc o aquecimento destas misturas à temperatura de $1400^{\circ} \mathrm{C}$. Para determinar o teor de enxofre de equilíbrio, foi simulado pelo Thermocalc, a reação entre as diferentes misturas e ferro gusa até a condição de equilíbrio. A utilização do Thermocalc, portanto, além de fornecer informações termodinâmicas do processo (\%S de equilíbrio, atividade dos componentes do metal e da escória) fornece dados que influenciam na cinética do processo (viscosidade, quantidade de líquidos e sólidos, e as fases presentes nas misturas), possibilitando uma melhor compreensão do processo.

\section{RESULTADOS E DISCUSSÃO}

\subsection{Resultados Experimentais}

A Figura 2 mostra a variação da concentração do enxofre com o tempo e a tabela 4 mostra os teores da \%S inicial, final, de equilíbrio e a eficiência de dessulfuração ( $\eta$ ).

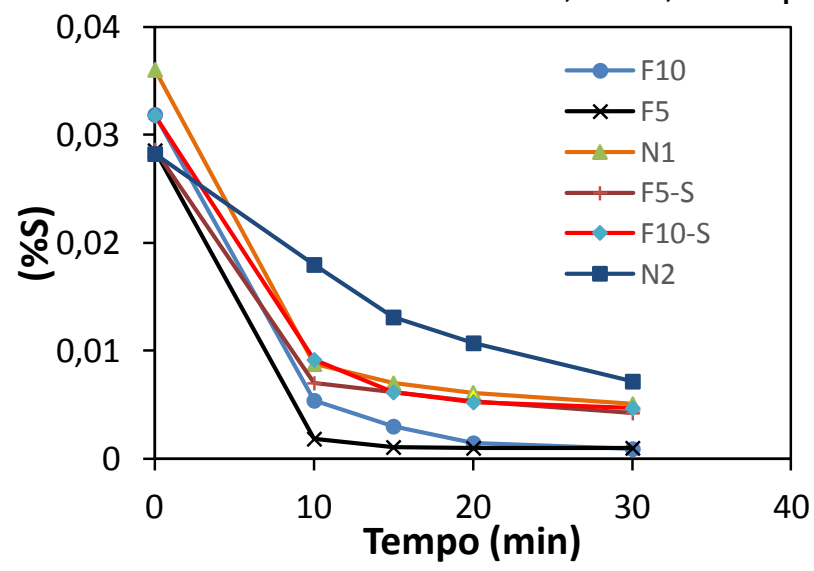

Tabela 4. Porcentagem de $S_{\text {incial }}, S_{\text {final }}$ e $S_{\text {eq. }}$.

\begin{tabular}{|c|c|c|c|c|} 
Mistura & \% $_{\text {inical }}$ & $\%_{\text {final }}$ & \% $_{\text {eq }}$ & $\eta(\%)$ \\
\hline F10 & 0,0319 & 0,0009 & $1,54.10^{-5}$ & 97,08 \\
\hline F5 & 0,0286 & 0,0010 & $1,57.10^{-5}$ & 96,43 \\
\hline N1 & 0,0360 & 0,0051 & $1,53.10^{-5}$ & 85,91 \\
\hline F5-S & 0,0286 & 0,0042 & $1,61.10^{-5}$ & 85,2 \\
\hline F10-S & 0,0318 & 0,0047 & $1,55.10^{-5}$ & 83,4 \\
\hline N2 & 0,0282 & 0,0072 & $1,56.10^{-5}$ & 74,58 \\
\hline
\end{tabular}

Figura 2. Variação da concentração de enxofre $[\%$ S] com o tempo 
A eficiência de dessulfuração (\%ๆ) foi calculado através da seguinte equação:

$$
\eta(\%)=\frac{\left[\% S_{i}\right]-\left[\% S_{f}\right\rfloor}{\left[\% S_{i}\right]} \times 100
$$

Onde $\left[\% S_{f}\right]$ é a concentração de enxofre final, e [\% $\left.S_{i}\right]$ é a concentração inicial de enxofre no ferro-gusa.

Pela tabela nota-se que a capacidade termodinâmica das escórias de remover enxofre é praticamente a mesma, pois os valores de \%Seq, foram praticamente iguais. Também pose-se nota que em nenhum experimento foi alcançado 0 equilíbrio.

Já pela Figura 2, observa-se que a velocidade de dessulfuração das misturas ( $\left.\frac{\Delta \% S}{\Delta t}\right)$, foi maior e constante até os primeiros 10 minutos de experimento, ocorrendo neste período a maior parte da dessulfurarão (de 50 a $90 \%$ ). A partir do $15^{\circ}$ minuto a velocidade de dessulfuração diminui de forma acentuada. Porém nenhuma das misturas alcançou a porcentagem de $S$ de equilíbrio.

Pela figura 2 nota-se que a mistura mais eficiente foi a mistura F10 seguida das misturas F5, N1, F5-S, F10-S e N2 respectivamente.

\subsection{Analise dos Modelos de $\Lambda, C_{s}$ e $L_{s}$ e da Eficiência de DeS}

Inicialmente, a capacidade de dessulfuração das misturas foi analisada através dos modelos capacidade de sulfeto $\left(C_{s}\right)$ e coeficiente de partição $\left(L_{s}\right)$ e da eficiência de $\operatorname{DeS}(\eta)$.

A Tabela 5 mostra os resultados encontrados.

Tabela 5. Valores de, $\Lambda, C_{s}$ e Ls e eficiência de dessulfuração das diferentes misturas usadas.

\begin{tabular}{cccccc}
\hline Mistura & $\boldsymbol{\Lambda}$ & $\mathbf{C s}_{\mathbf{s}}$ & $\mathbf{L s}_{\mathbf{s}}$ & $\Delta \% \mathbf{S}$ & $\eta(\%)$ \\
\hline F10 & 0,930 & 1,2081 & 8420 & 0,0309 & 97,08 \\
F5 & 0,953 & 1,2354 & 14955 & 0,0276 & 96,43 \\
N1 & 0,947 & 1,2285 & 13019 & 0,0309 & 85,91 \\
F5-S & 0,927 & 1,2043 & 7731 & 0,0244 & 85,2 \\
F10-S & 0,905 & 1,1813 & 4466 & 0,0271 & 83,4 \\
N2 & 0,920 & 1,1964 & 6448 & 0,0211 & 74,58 \\
\hline
\end{tabular}

Pela analise da da Tabela 5,nota-se que as misturas dessulfurantes do sistema $\mathrm{CaO}_{-} \mathrm{CaF}_{2}$ (F5 e F10) tiveram as maiores eficiencia de dessulfuração, com 96,43\% e $97,08 \%$, respectivamente. No entanto, a adição de duas vezes a quantidade de fluorita na mistura F10 com relação amistura F5, não mostrou ter muita influência sobre a eficiência, com um aumento de apenas $0,65 \%$.

A adição de 2 e $4 \%$ de $\mathrm{SiO}_{2}$ nestas misturas dimniue sua eficiencia em torno de $12 \%$.

Através dos resultados dos modelos termodinâmicos mostrados na Tabela 5, podese notar que as misturas com maior capacidade termodinâmica de remover 0 enxofre do ferro-gusa são a F5 e a N1, as quais apresentaram maiores valores de $\Lambda$ e, consequentemente, maiores valores de $\mathrm{C}_{\mathrm{s}}$ e $\mathrm{L}_{\mathrm{s}}$.

Os melhores resultados para as misturas $\mathrm{F} 5$ e $\mathrm{N} 1$ pode ser justificado pela maior concentração de $\mathrm{CaO}$ nas mesmas. A Figura 3 mostra a relação entre esses parâmetros e a eficiência de DeS. 

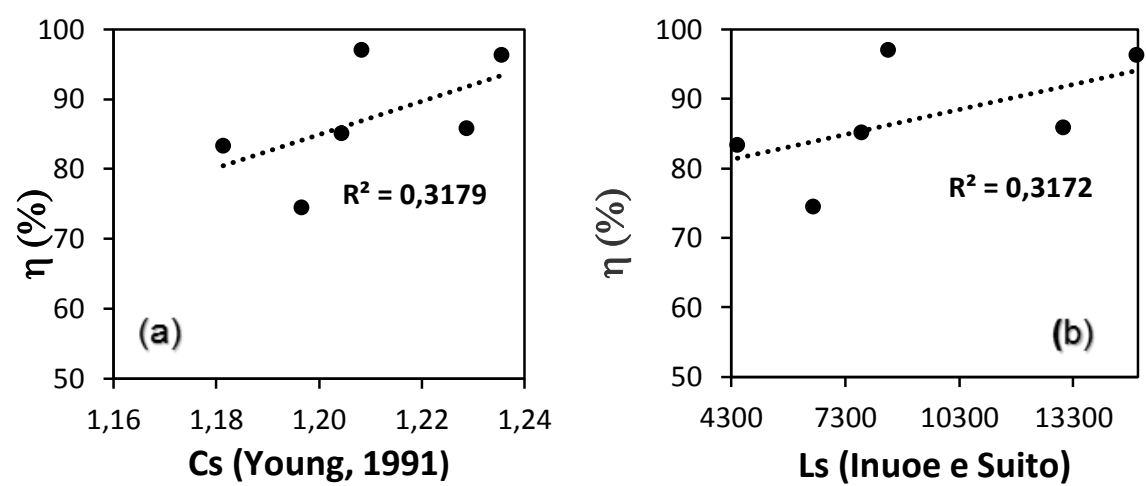

Figura 3 Relação entre a) Cs e b) Ls com a eficiência de dessulfuração obtida nos tetes.

A Figura 3 mostra que quanto maior $\wedge$, CS e LS, maior a eficiência. Porém, de acordo com a Figura, note-sa que não há uma boa correlação $\left(R^{2}\right)$ entre os modelos termodinâmicos da literatura e a eficiência obtida em testes experimentais. Esta correlação $\left(R^{2}\right)$ foi inferior a 0,4 .

Assim, as simulações feitas pelo Thermocalc podem se tornar uma alternativa para avaliar e identificar as variáveis que afetam a eficiência de dessulfuração das diferentes misturas, como percentual de enxofre de equilíbrio,fases presentes e percentual de sólidos e líquidos.

\subsection{Simulação Aquecimento das misturas dessulfurantes através do ThermoCalc}

A Tabela 6 mostra o teor de enxofre de equilíbrio, as fases e percentagem de sólidos e líquido na mistura a $1.400^{\circ} \mathrm{C}$, determinados pelo Thermocalc.

Tabela 6. Valores da \%Seq e porcentagem de fases formadas nos diferentes experimentos determinados pelo Thermocalc

\begin{tabular}{|c|c|c|c|c|c|c|c|}
\hline \multirow[b]{2}{*}{ Mistura } & \multirow[b]{2}{*}{$\eta(\%)$} & \multirow[b]{2}{*}{ \%Liquido } & \multicolumn{3}{|c|}{ Fases sólidas (\%) } & \multirow[b]{2}{*}{$\begin{array}{l}\text { C3S+ } \\
\text { 3CA. }\end{array}$} & \multirow[b]{2}{*}{$\% S_{\text {Eq. }}\left(\mathbf{T}^{\star}\right)$} \\
\hline & & & $\mathrm{CaO}_{(\mathrm{s})}$ & $\mathrm{C}_{3} \mathrm{~S}$ & $\mathrm{C}_{3} \mathrm{~A}$ & & \\
\hline F10 & 97,0 & 16,82 & 79,6 & 3,6 & - & 3,6 & $1,54.10^{-5}$ \\
\hline F5 & 96,43 & 9,2 & 85,1 & 5,7 & - & 5,7 & $1,57.10^{-5}$ \\
\hline N1 & 85,91 & 2,56 & 77,2 & 16,8 & 3,5 & 20,2 & $1,53 \cdot 10^{-5}$ \\
\hline F5-S & 85,2 & 8,8 & 74,5 & 16,6 & - & 16,6 & $1,61 \cdot 10^{-5}$ \\
\hline F10-S & 83,4 & 16,8 & 69 & 14,2 & - & 14,2 & $1,55.10^{-5}$ \\
\hline N2 & 74,4 & 4,8 & 64 & 25,2 & 6,4 & 31,6 & $1,56 \cdot 10^{-5}$ \\
\hline
\end{tabular}

$\mathrm{CaO}_{\mathrm{s}}=$ Porcentagem de $\mathrm{CaO}$ sólido que não foi usado para formar $\mathrm{C}_{3} \mathrm{~S}$ e ou $\mathrm{C}_{3} \mathrm{~A} ; \mathrm{C}_{3} \mathrm{~S}=3 \mathrm{CaO}_{\mathrm{SiO}}$; $\mathrm{C}_{3} \mathrm{~A}=3 \mathrm{CaO} \cdot \mathrm{Al}_{2} \mathrm{O}_{3}$

A Tabela 6 mostra que, em todas as misturas ocorreu uma redução da concentração de $\mathrm{CaO}$ sólido quando foi feita a simulação do aquecimento até $1400^{\circ} \mathrm{C}$. Isto ocorreu porque parte do $\mathrm{CaO}$ das msituras foi usado para formar 3CaO.SiO2 e / ou 3CaO.Al2O3 e parte foi usado para formar a fase líquida

A Tabela 6 mostra também que o teor de enxofre de equilíbrio obtido em ambas as misturas são quase os mesmos. Portanto, pode concluir-se que, termodinamicamente, as misturas tem aproximadamente a mesma capacidade para remover o enxofre do ferro-gusa.

No entanto, este resultado não foi encontrado nos testes experimentais, de acordo com a Figura 2 e a Tabela 6, que msotram que o rendimento de cada experimento 
foi diferente. Isto pode ter ocorrido devido à quantidade de fase líquida presente nas misturas e a formação de compostos sólidos, tais como silicato tricálcico $\left(3 \mathrm{CaO} . \mathrm{SiO}_{2}\right)$ e aluminato tricálcico $\left(3 \mathrm{CaO} \cdot \mathrm{Al}_{2} \mathrm{O}_{3}\right)$ [6]. A quantidade de fase líquida e de compostos sólidos, tais como silicato tricálcico $\left(3 \mathrm{CaO} . \mathrm{SiO}_{2}\right)$ e $3 \mathrm{CaO} .\left(\mathrm{Al}_{2} \mathrm{O}_{3}\right)$ infleuciam a cinética da dessulfuração e, desta forma, o teor final de enxofre.

A simulação de aquecimento das misturas através do Thermocalc nos fornece estas informações como mostra a Tabela 4. Pode-se notar pela tabela que, as misturas F10 e F5 mostram uma quantidade maior de líquido do que as outras misturas. Além disso, estas misturas mostraram uma menor formação dos compostos que podem limitar o transporte de massa de enxofre, silicato tricálcico $\left(3 \mathrm{CaO}^{\left.-\mathrm{SiO}_{2}\right)}\right.$ e aluminato tricálcico $\left(3 \mathrm{CaO} \cdot \mathrm{Al}_{2} \mathrm{O}_{3}\right)$. Isto ocorreu, porque estas misturas são as que tem maior porcentagem de $\mathrm{CaF} 2$ e menor porcentagem de $\mathrm{SiO}_{2}$.

Portanto, pela simulação do aquecimento das misturas dessulfurantes a $1400^{\circ} \mathrm{C}$ é de esperar que, cineticamente, as misturas F5 e F10 apresentem melhores resultados do que as outras

A Figura 5 mostra a relação entre estas variáveis porcentagem de liquido, porcentagem de $\mathrm{CaO}$ solido e das fases $3 \mathrm{CaO} . \mathrm{SiO}_{2} 3 \mathrm{CaO} \cdot \mathrm{Al}_{2} \mathrm{O}_{3}$ na eficiência de dessulfuração.
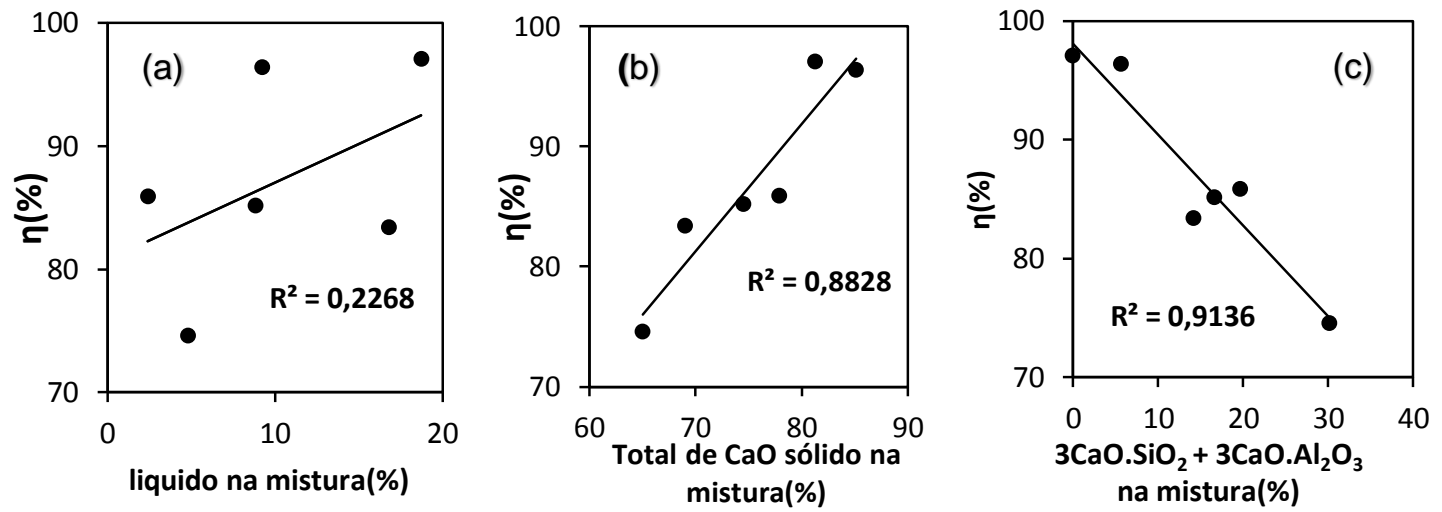

Figura 5 Influência da a) porcentagem de líquido, b) porcentagem de $\mathrm{CaO}$ sólido e c) fases $3 \mathrm{CaO} \cdot \mathrm{SiO}_{2}+3 \mathrm{CaO} \cdot \mathrm{Al}_{2} \mathrm{O}_{3}$ na eficiência de dessulfuração.

A Figura 5 mostra que quanto maior foi a quantidade de líquido de escória e menor a formação das fases $3 \mathrm{CaO}$. $\mathrm{SiO}_{2}$ e $3 \mathrm{CaO} . \mathrm{Al}_{2} \mathrm{O}_{3}$ nas misturas, maior foi a eficiência.

A maior relação encontrada $\left(R^{2}=0,91\right)$ foi entre a eficiência de dessulfuração e a soma das fases $3 \mathrm{CaO} . \mathrm{SiO}_{2}$ e $3 \mathrm{CaO} \cdot \mathrm{Al}_{2} \mathrm{O}_{3}$, como mostrada a figura 5 (d). De acordo com a figura quando maior a presença destas fases, menor é a eficiência da dessulfuração, uma vez que estas se formam em torno das partículas de $\mathrm{CaO}$, impedindo o transporte de massa do enxofre até ela.

Já a relação entre a influência de dessulfuração e a quantidade de fase liquido formado nas misturas foi baixa $\left(R^{2}=0,227\right)$. Isto é um indicativo de que nos processos estudados no presente trabalho, a fase liquida formada não tem influência direta na reação de dessulfuração, agindo somente na dissolução de parte da fase sólida formada.

Como a dessulfuração ocorre pela reação direta entre as partículas de $\mathrm{CaO}$ e o enxofre no metal liquido, é preciso levar em consideração as partículas de $\mathrm{CaO}$ que estão efetivamente disponíveis para reagir. Como as fases $3 \mathrm{CaO} . \mathrm{SiO}_{2}$ e $3 \mathrm{CaO} \cdot \mathrm{Al}_{2} \mathrm{O}_{3}$ se formam em torno das partículas de $\mathrm{CaO}$, é preciso também verificar a influência conjunta destes parâmetros na eficiência de dessulfuração. Para isto foi criado o parâmetro \% $\mathrm{CaO}$ livre (\%CaO $)$ de acordo com a equação 11. 
$\% \mathrm{CaO}_{\mathrm{L}}=\left(\% \mathrm{CaO}_{s}\right)-\left(\% 3 \mathrm{CaO} \cdot \mathrm{SiO}_{2}+\% 3 \mathrm{CaO} \cdot \mathrm{Al}_{2} \mathrm{O}_{3}\right)$

Assim, a percentagem de $\mathrm{CaO}\llcorner$ foi definida como a percentagem de $\mathrm{CaO}$ sólido menos a percentagem de $3 \mathrm{CaO} . \mathrm{SiO}_{2}$ e $3 \mathrm{CaO} \cdot \mathrm{Al}_{2} \mathrm{O}_{3}$ como mostrado Equação 11.

Portanto, $\mathrm{CaO}$ livre é a quantidade de $\mathrm{CaO}$ que está efetivamente disponível para reagir com o enxofre contido no ferro gusa.

A Tabela 6 mostra os valores da \%CaO obtidos, e a Figura 6 a influência da \%CaO na eficiência de dessulfuração.

Tabela 6. Valores de $\mathrm{CaO}$ livre

\begin{tabular}{lll}
\hline Mistura & $\eta(\%)$ & \%CaO \\
& & \\
\hline F10 & 97,1 & 79,9 \\
F5 & 96,4 & 76,0 \\
N1 & 85,9 & 57,25 \\
F5-S & 85,2 & 57,9 \\
F10-S & 83,4 & 53,8 \\
N2 & 74,6 & 32,1 \\
\hline
\end{tabular}

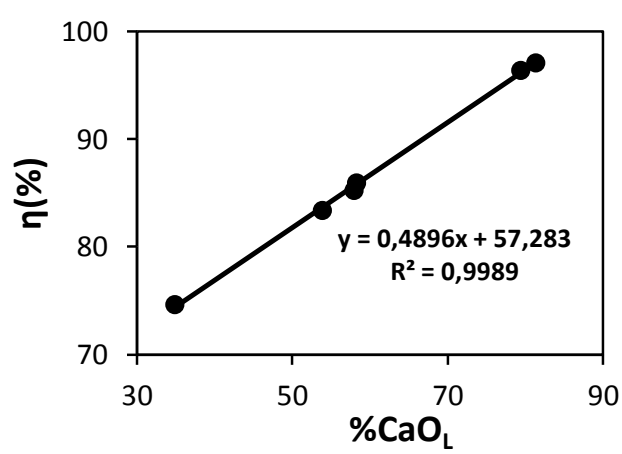

Figura 6. Influência do CaO livre na eficiência de dessulfuração.

A Figura 6 mostra que a quanto maior a porcentagem de $\mathrm{CaO}$ nas misturas maior será aeficiencia de DeS. Mostra também que acorrelação entre a \% $\mathrm{CaO}$ e a eficiencia é praticamente 1.

Pode-se então estabelecer um modelo para prever a eficiência de dessulfuração (n) de ferro gusa com misturas a base de $\mathrm{CaO}-\mathrm{CaF}_{2}, \mathrm{SiO}_{2}, \mathrm{Al}_{2} \mathrm{O}_{3}$, e $\mathrm{Na}_{2} \mathrm{O}$ com base na $\% \mathrm{CaO}_{\mathrm{L}}$, através da equação da reta obtida no gráfico gerado pela relação entre $\eta \times \% \mathrm{CaO}$.

Porém, esta relação vale desde que sejam respeitadas as mesmas condições de temperatura, e agitação do banho. Para o caso do presente trabalho pode-se estabelecer o seguinte modelo:

$$
\begin{aligned}
& \eta(\%)=0,4896\left(\% \mathrm{CaO}_{\mathrm{LS}}\right)+57,28 \\
& \eta(\%)=0,4896\left[\left(\% \mathrm{CaO}_{\mathrm{S}}\right)-\left(\% 3 \mathrm{CaO} \cdot \mathrm{SiO}_{2}+\% 3 \mathrm{CaO} \cdot \mathrm{Al}_{2} \mathrm{O}_{3}\right)\right]+57,28
\end{aligned}
$$

Estes resultados levam à conclusão de que realmente os compostos sólidos $3 \mathrm{CaO} . \mathrm{SiO}_{2}$ e $3 \mathrm{CaO} . \mathrm{Al}_{2} \mathrm{O}_{3}$ diminuiem a eficiencia de dessulfuraçào do $\mathrm{CaO}$ devido ao fato de se formarm em torno dele.

A formação destes compostos, diminui a área de $\mathrm{CaO}$ eficaz disponível para reagir com o enxofre, o que diminui a eficiencia da reação de dessulfuração, como mostrado na Equação 8.

Como termodinamicamente as misturas apresentam quase a mesma eficiência, a cinética torna-se um fator importante para melhorar a dessulfuração.

Também pode se afirmar pela análise da Figura 6 que o parâmetro \%CaOL, tem relação direta com a eficiência de dessulfuração, sendo a correlação entre as variáveis igual a 1.

Com base na equação 13 e nas Tabelas 2 e 6, pode-se notar que uma maneira de aumentar o parametro $\% \mathrm{CaO}\left\llcorner\right.$ é diminuindo a porcentagem de $\mathrm{SiO}_{2}$ nas matérias primas usadas. 
Com base nisto, pode-se determinar previamnete, se uma mistura com matérias primas contendo maiores ou menores teores de $\mathrm{SiO}_{2}$ vai ter a eficiencia de dessulfuração pretendida.

Outra forma de aumentar o parametro $\% \mathrm{CaO}_{\mathrm{L}}$ é atarvés da utilização da fluorita, que pode diminuir a porcentagem das fases $3 \mathrm{CaO} . \mathrm{SiO}_{2}$ e $3 \mathrm{CaO} . \mathrm{Al}_{2} \mathrm{O}_{3}$, como ocorreu com as misturas F10 e F5.

Outra posibilidade de aplicação do metodo que resultou na Equação 11, é usar no lugar da porcentagem dos constituites a massa destes. Com isto popde-se detrminar previamente, se uma massa menor ou maior de mistura pode também alcançar a eficiencia desejada.

Assim, é possível afirmar que a melhor mistura dessulfurante de ferro gusa é a que apresentar as seguintes propriedades quando adicionada ao banho:

1 Maior concentração de $\mathrm{CaO}$ sólido na escória;

2 Menor formação dos compostos silicato tricálcico $\left(3 \mathrm{CaO} . \mathrm{SiO}_{2}\right)$ e aluminato tricálcico $\left(3 \mathrm{CaO} \cdot \mathrm{Al}_{2} \mathrm{O}_{3}\right.$.

Isto porque quanto maior a quantidade de fase líquida, menor será a formação de fases sólidas $3 \mathrm{CaO} . \mathrm{SiO}_{2}$ e $3 \mathrm{CaO} \cdot \mathrm{Al}_{2} \mathrm{O}_{3}$. Estas fases são formadas em torno da partícula $\mathrm{CaO}$ sólido, impedindo a transferência do $\mathrm{S}$ no metal até que a partícula de $\mathrm{CaO}$.

Com base nestes resultados, pode-se afirmar que o uso de Thermocalc permite uma melhor análise e previsão da eficiencia de dessulfuração das misturas usadas. Isto possibilita uma avaliação mais precisa de qual será a melhor mistura desulfurante para ser utilizado em processos industriais que os modelos de Cs e Ls. Além disto, o uso do Thermocalc permitiu o desenvolvimento de um modelo para prever a eficiencia de desfosofração das misturas a base de $\mathrm{CaO}$.

\section{CONCLUSÕES}

Com base nas condições e nos resultados obtidos no presente estudo, pode-se concluir que:

- As misturas F5 e F10, apresentaram uma maior eficiência de dessulfuração, com $96,43 \%$ e $97,08 \%$, respectivamente;

- A adição de duas vezes a quantidade de fluorita na mistura CF10 não mostrou muita influência sobre a eficiência de dessulfuração, com um aumento de apenas $0,65 \%$ na eficiência;

- As misturas que apresentaram maior eficiência foram os que apresentaram o maior percentual de $\mathrm{CaO}$;

-Nos processos estudados no presente trabalho a fase liquida formada não tem influência direta na reação de dessulfuração, agindo somente na dissolução de parte das fases sólidas formadas;

- Os parâmetros termodinâmicos Cs e Ls podem levar a conclusões errôneas sobre a eficiência de dessulfuração de diferentes misturas;

- O parâmetro $\mathrm{CaO}$, tem relação direta com a eficiência de dessulfuração, sendo a correlação entre as variáveis iguais a 1.Com isto pode-se estabelecer para as condições do presente trabalho o seguinte modelo para a eficiência de dessulfuração:

$\eta(\%)=0,4896\left[\left(\% \mathrm{CaO}_{\mathrm{S}}\right)-\left(\% 3 \mathrm{CaO} \cdot \mathrm{SiO}_{2}+\% 3 \mathrm{CaO} \cdot \mathrm{Al}_{2} \mathrm{O}_{3}\right)\right]+57,28$

- Com a utilização do parâmetro \%CaO pode -se prever a influência da porcentagem de $\mathrm{SiO}_{2}$, de $\mathrm{CaF}_{2}$ e da massa das misturas na eficiência de 
dessulfuração. Este parâmetro é mais eficiente que a utilização de Cs e Ls para prever qual mistura será mais eficiente;

- O ThermoCalc mostrou ser uma ferramenta precisa na previsão da eficiência das misturas dessulfurantes.

\section{REFERÊNCIAS}

1 ANDERSON, M.; JÖNSSON, P.; HALLBERG, M. Optimization of ladle slag composition by application of sulphide capacity model. Ironmaking and Steelmaking, n. 4, v. 27, p. 286-293, 2000.

2 TURKDOGAN, E.T. Fundamentals of Steelmaking. 1. ed. London: The Institute of Materials, 1996. 331p.

3 VISSER, H.; BOOM, R. Advanced Process Modelling of Hot Metal Desulphurisation by Injection of $\mathrm{Mg}$ and $\mathrm{CaO}$. ISIJ International, n. 12, v. 46, p. 1771-1777, 2006.

4 OHYA, T. Desulfurization of Hot Metal with Burnt Lime. Steelmaking Proceedings. V. 60, 1977.

5 MITSUO, T.; SHOJI, T.; HATTA, Y.; ONO, H.; MORI, H.; KAI, T. Improvement of desulfurization by addition of aluminum to hot metal in the lime injection process. Transactions of the Japan Institute of Metals, n. 12, v. 23, p. 768-779, 1982.

6 NIEDRINGHAUS, J. C.; FRUEHAN, R. J. Reaction Mechanism for the CaO-Al and $\mathrm{CaO}-\mathrm{CaF}_{2}$ Desulfurization of Carbon-Saturated Iron. Metallurgical Transactions $\mathrm{B}, \mathrm{v}$. 19B, p. 261-268, 1988.

7 SOSINSKY D.J., SOMMERVILLE I.D. The Composition and Temperature Dependence of the Sulfide Capacity of Metallurgical Slags. Metallurgical Transactions B, v. 17B, p. 331-337, 1986.

8 YOUNG, R.W. Use of the optical basicity concept for determining phosphorus and sulphur slag/metal partitions. London: British Steel, 1991. 115p. cap 4, p. 7.

9 INOUE, R.; SUITO, H. Sulfur Distribution between Carbon-Saturated Iron Melt and Na2O -SiO2 Slags. Transactions ISIJ, v. 22, p. 514-523, 1982.

10 YOSHINORI, T., NOBUO, S., SESHADRI, S. Sulphide Capacities of $\mathrm{CaO}-\mathrm{Al}_{2} \mathrm{O}_{3}-\mathrm{SiO}_{2}-$ MgO-MnO Slags in the Temperature Range 1673-1773 K. ISIJ International, v. 49, n. 2, p. 156-163, 2009. 